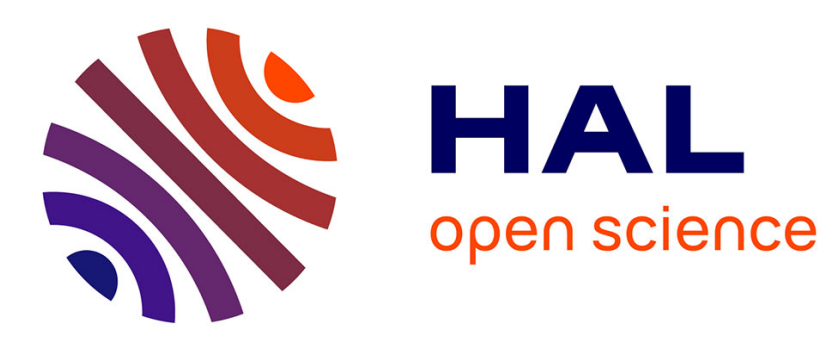

\title{
Le dopage dans le cyclisme professionnel: accusations, confessions et dénégations
}

\author{
Patrick Trabal, Pascal Duret
}

\section{To cite this version:}

Patrick Trabal, Pascal Duret. Le dopage dans le cyclisme professionnel: accusations, confessions et dénégations. STAPS : Revue internationale des sciences du sport et de l'éducation physique, 2003, 60 (1), pp.59. 10.3917/sta.060.0059 . hal-03016963

\section{HAL Id: hal-03016963 \\ https://hal.parisnanterre.fr/hal-03016963}

Submitted on 20 Nov 2020

HAL is a multi-disciplinary open access archive for the deposit and dissemination of scientific research documents, whether they are published or not. The documents may come from teaching and research institutions in France or abroad, or from public or private research centers.
L'archive ouverte pluridisciplinaire $\mathbf{H A L}$, est destinée au dépôt et à la diffusion de documents scientifiques de niveau recherche, publiés ou non, émanant des établissements d'enseignement et de recherche français ou étrangers, des laboratoires publics ou privés. 


\title{
Le dopage dans le cyclisme professionnel : accusations, confessions et dénégations
}

\author{
Patrick Trabal ${ }^{1}$ et Pascal Duret ${ }^{2}$ \\ 1. Univ. Paris X, Lab. Sport \& Culture, 200 av. de la République - 92001 Nanterre Cedex \\ 2. Univ. de la Réunion, CURAPS, 117 rue du Général Ailleret - 97430 Le Tampon
}

Résumé: Le but de cet article est d'étudier la question du dopage en mobilisant les apports de la sociologie pragmatique. En étudiant symétriquement les positions des différents protagonistes et en les prenant au sérieux, cette approche se propose de rendre compte de l'importance des fondements politiques et moraux qui guident les acteurs. L'affaire Festina du Tour de France 1998 constitue l'objet de l'article; le corpus, construit à partir de cinq livres (publiés par les principaux acteurs) fut analysé avec le logiciel Prospéro. Nous avons notamment étudié les procédés mobilisés par les protagonistes pour convaincre, les « grandeurs » auxquelles ils se réfèrent pour défendre leurs conceptions du légitime et de l'illégitime, ainsi que leurs points de vue politiques sur l'avenir du cyclisme. La « figure » du récit à la première personne, par laquelle le locuteur se présente comme un témoin relatant des faits sous la forme d'une confession, apparaît comme la plus efficace des stratégies rhétoriques. Nous avons distingué cinq « grandeurs » auxquelles les protagonistes se réfèrent quand ils évoquent le dopage dans le cyclisme. Cette pluralité permet de comprendre les tensions du conflit ainsi que les oppositions dans leurs propositions politiques pour sortir de la crise.

Mots-clef: Sociologie, dopage, cyclisme, justice, preuve.

\section{Doping in professional cycling : charges, confessions and denials}

\begin{abstract}
The aim of this paper is to study doping bringing-in pragmatic sociology. By avoiding costing blames, in studying the positions of different protagonists, this sociology attaches great importance to the political and moral foundations which guide social actors. We are studying doping in the Tour de France 1998; Five books, written by the principal actors concerned in this affair, lead us to build a corpus, analyzed by the software "Prospéro". We are working on the process used by the protagonists to persuade people, on the plurality of "greatnesses" they refer in their conception of what it is legitimate and what it is not, and the political points of view concerning the future of cycling. Among different rhetorical strategies, to use the first person singular in introducing oneself as a confessed witness, seems very effective. We have made out five "greatnesses" used by the protagonists to justify their points of view; this plurality allows us to understand tension and opposition in their political suggestions to emerge from this crisis.
\end{abstract}

Keywords : Sociology, Doping, professional cycling, justice, proof 


\section{INTRODUCTION}

La capacité du sport à concilier justice et concurrence repose sur l'obligation d'établir des épreuves où les premiers sont toujours les meilleurs sans tricher. Pour que « la justice devienne le produit de la concurrence » (Ehrenberg, 1991 : 18), l'épreuve sportive doit impérativement garantir l'égalité des chances entre concurrents. A cette condition seulement, il peut y avoir exaltation du classement méritocratique, célébration du vainqueur et accord sur sa grandeur. Le dopage trouble cette exigence de justice quand il passe inaperçu comme quand une « affaire » éclate venant contrarier la construction du mythe de la « juste inégalité » sportive. Mais le sens des indignations n'est pas univoque. Certaines sources dénoncent l'atteinte à « l'esprit coubertinien » du sport, d'autres les mauvais traitements réservés aux sportifs par leurs entraîneurs ou au contraire par les « policiers », d'autres enfin le pouvoir excessif des médias. Les lieux de l'indignation eux-mêmes se diversifient; les affaires liées au dopage marquent en particulier une transformation importante dans la manière de rendre compte des événements sportifs puisque les journalistes qui s'y attachent le font souvent hors des rubriques spécialisées. Mis en accusation les sportifs, les soigneurs et les organisateurs, sont de leur côté tenus de se justifier en ouvrant une controverse sur la qualification de la faute, son juste traitement et sa réparation. Se faisant, l'affaire opère un déplacement de «l'épreuve de justice » en empruntant à des catégories de jugement extra-sportives. La controverse produit donc une recomposition d'ensemble de l'épreuve dont l'enjeu est justement l'imposition de nouvelles règles à même de déterminer la distribution des rôles des différents acteurs. Les « vérités » relatives de chacun des protagonistes ne font pas que s'appuyer sur des discours normatifs reprenant la simple opposition entre ce qui est permis et ce qui ne l'est pas. Elles renvoient à une morale personnelle, même si elle a besoin de l'approbation de l'opinion, qui questionne ce qui est légitime ou non, en rediscutant les frontières entre le glorieux et le honteux.

Pour analyser comment se déplacent ces lignes de démarcations nous avons pris pour terrain le Tour de France 1998. Rappelons en, à grands traits, le déroulement. A la suite de l'arrestation d'un soigneur (Willy Voet) qui précède celle du directeur d'équipe (Bruno Roussel), les coureurs de l'équipe Festina, sans avoir été reconnu fautifs par les contrôles de l'institution sportive, vont être exclu de l'épreuve. Le plus médiatisé et le plus populaire d'entre eux, Richard Virenque, niera systématiquement et durablement s'être dopé. S'en tenir au rapport judiciaire clôt immédiatement le débat car il s'énonce comme un constat: le soigneur qui a transporté les produits est assimilé à un trafiquant, les coureurs à des consommateurs de drogues. Mais l'incertitude sur les statuts de chacun des acteurs est entretenue par les journalistes, leurs avocats, les spectateurs et les coureurs eux-mêmes. Est-ce Voet qui a entraîné la ruine des Festina? Roussel a-t-il lâché les coureurs en avouant? Quel statut donner à l'aveu de certains coureurs? Autant de questions morales qui permettent l'étude de ce moment d'incertitude que constitue l'épreuve dans laquelle nos acteurs expriment et confrontent leurs conceptions de la légitimité, de l'honneur, de l'inadmissible et du honteux.

\section{MOBILISER LA SOCIOLOGIE PRAGMATIQUE}

Le modèle théorique de référence, souvent qualifié de « sociologie pragmatique », s'inscrit dans la tradition des sociologies de l'action. S'il est difficile de prétendre le résumer en quelques lignes, il convient cependant de rappeler quelquesunes de ses caractéristiques en raison ses usages relativement peu fréquents dans les recherches sur l'épreuve sportive.

A la suite des travaux des sociologues américains, notamment de Garfinkel, Boltanski s'intéressa dès 1984 (Boltanski et al., 1984) à la façon dont sont dénoncées des injustices. Dans cette perspective, il ne s'agit plus de rendre compte d'une situation, en recensant préalablement les positions des protagonistes comme des facteurs dispositionnels expliquant les attitudes des « agents ». Il s'agit ici d'étudier précisément comment ils se déterminent en leur accordant le statut «d'acteurs ». Du coup, 
on chercha à décrire finement les « actions » qu'ils entreprennent, en nous efforçant de ne pas les qualifier préalablement. Ce projet opérait une double rupture. D'une part avec les modèles visant à décoder la rationalité des agents prioritairement dans le jeu des structures sociales. Et, d'autre part, à se démarquer tout autant des approches ramenant, à la manière de l'ethnométhodologie, les faits sociaux à de simples effets de situations, toujours rejouables au gré des interactions des acteurs (« members »). A ce titre, on ne peut en rendre compte de cette sociologie sans se référer à ces deux courants dont elle cherche à se démarquer. Il s'agit de dégager des catégories du jugement moral que mobilisent les personnes, en bonne partie indépendamment à leur position sociale, sans pour autant céder au recours aux statistiques car elles supposent la construction de regroupements (Boltanski et Thévenot, 1991, p. 12). Or ceux-ci posent problème moins dans leur adéquation à la réalité (toujours discutable en vue d'une révision suite à l'évaluation de leur pertinence) que dans le rôle du chercheur lorsqu'il les constitue. En classifiant, en regroupant des réalités différentes sous un même nom, en introduisant de la mesure là où la situation se déroule sans référence à une métrologie, en un mot en objectivant, la sociologie peut déformer la réalité dont elle cherche à rendre compte, non pas par les faiblesses des nomenclatures mobilisées par le chercheur, mais par son activité même. Son travail produit notamment comme effet, un découpage du social en structures, intrinsèquement liées de fait au travail du sociologue, une division entre des groupes, qui pourtant communiquent entre eux et tentent souvent de surmonter leur opposition en négociant, en s'accordant, et en créant du lien. Face une sociologie qui ne cesse de diviser l'espace social pour s'interroger sur les forces en présence, il s'agit de construire des modèles rendant compte des accords, des contrats moraux et des principes de justice auxquels les acteurs se réfèrent, en refusant délibérément de les qualifier préalablement. L'exigence prag- matique peut donc se formuler ainsi: dans la tradition de Charles Sanders Peirce ou de William James qui s'attachent à montrer combien les questions pratiques sont déterminantes dans les actions et les conceptions des individus, il faut s'employer à partir non pas de « dispositions acquises » ou de « manières durables d'être ou de faire qui s'incarnent dans les corps » (Bourdieu, 1984 : 29), mais des contraintes pesant sur les acteurs. Il s'agit essentiellement de recenser et de décrire leurs actions pour étudier comment ils subissent des impératifs et comment ils les gèrent. Cela ne signifie pas que nous souhaitons évacuer les rapports de forces ${ }^{1}$ et les jeux de dominations entre des groupes sociaux. Nous refusons simplement de les considérer comme des préalables à l'investigation sociologique. En effet, les acteurs nous semblent largement capables d'indiquer les jeux de pouvoir et les épreuves de forces dans lesquels ils sont engagés; nous préférons partir des conséquences liées aux structures sociales plutôt que des les postuler. Les acteurs sont d'ailleurs tout aussi capables de rappeler les contrats qui les lient lorsqu'ils invoquent des principes de légitimité. Il faut renoncer, et en cela suivre Francis Chateauraynaud (1991), à réduire les jeux sociaux à des épreuves de force, pas plus qu'à des épreuves de légitimité. En revanche, il nous faut rendre compte du passage de la force à la légitimité (comme de l'inverse).

En ce sens, le cadre privilégié permettant d'étudier ces déplacements reste la dispute. La diversité de ses formes (simple polémique, contentieux, scandale, affaire) offre autant de cas permettant d'analyser ses différentes phases. Il s'agit de recenser comment sont mobilisées les différentes ressources en déployant la carte des réseaux, des accords existants, des contrats et de tout autre type de liens, et comment s'exerce le jugement. En décrivant les contraintes pesant sur les acteurs, les principes de justices, et les grandeurs morales auxquels ils se réfèrent, il est alors possible de comprendre les épreuves dans lesquelles ils sont engagés, c'est-à-dire ces moments d'incertitudes à l'issue desquelles les situa-

1. On ne peut ignorer par exemple que le succès du sport tient pour une bonne part à son incessante adaptation aux modifications structurelles de l'offre médiatique (Marchetti, 1998), pas plus qu'on ne peut ignorer les rapports concurrentiels entre groupe de presse. 
tions se trouvent reconfigurées. Enfin, pour clore une controverse, sont souvent définis de nouveaux accords; il s'agit alors de décrire finement les processus par lesquels ces nouveaux contrats sont négociés, acceptés par les différents protagonistes et mis en œuvre.

La sociologie pragmatique s'intéresse donc particulièrement aux processus d'attribution des responsabilités ainsi qu'aux disputes car ils permettent d'étudier les procédés qui mènent aux accords entre les acteurs. Mais elle veille à ne pas les qualifier: recenser leurs intérêts et les statuts qu'ils ont à défendre, revient d'une part à négliger le principe de neutralité axiologique (la désignation des victimes et des responsables hypothèque sérieusement la consigne wéberienne), et d'autre part, à figer la situation. Or les rôles joués par les acteurs évoluent et sont multiples (Dodier, 1991, Bénatouill, 1999). Du coup, on renoncera à procéder comme le juriste ou le journaliste et plutôt que d'attribuer des responsabilités à des fins de classification, on étudiera les processus par lesquels l'ensemble des acteurs, s'indignent, dénoncent, accusent, justifient, étayent leurs preuves, condamnent. L'on repère alors des épreuves de légitimité (ou de « grandeur» - Boltanski et Chiapello, 1999), dans lesquelles la défaillance renvoie à une dimension morale, au sens où l'être moral est celui qui tient ses engagements. Ainsi, cette sociologie se propose de considérer des acteurs, qui sensibles aux injustices, s'avèrent capables surmonter les épreuves de la vie quotidienne et de mobiliser des compétences plurielles leur permettant de se faire un jugement, de développer des valeurs morales et d'exprimer des visées politiques.

La question du dopage constitue un exemple intéressant pour éprouver ce modèle. D'une part, elle permet de montrer comment les acteurs se jugent et s'entrejugent en portant des critiques sur leurs propres manières d'agir en s'appuyant moins sur des codes moraux externes que sur des engagements personnels passés entre eux comme avec les employeurs, et le public. D'autre part, elle occupe suffisamment l'espace public pour que le chercheur puisse recueillir les prises de position des différents protagonistes, recenser leurs lec- tures des évènements, les inférences produites, leurs jugements... Notre démarche présuppose que les acteurs sociaux soient largement capables du travail d'imputation de responsabilité. Or si le silence semble régir le monde sportif dans son rapport au dopage, nos acteurs n'hésitent pas à s'exprimer publiquement pour exposer à la vindicte publique certaines pratiques, et pour se soumettre à des contraintes de justification. Il convient alors justement de suivre ses justifications sans s'attacher à l'une d'entre elle en cherchant à la défendre contre les autres, mais au contraire en conservant neutralité et traitement symétrique. Cette tâche n'est pas toujours aussi facile qu'elle y paraît. Il est par exemple d'autant moins aisé de prendre la position de Richard Virenque au sérieux que les guignols de l'info n'ont de cesse de le disqualifier l'assimilant à un idiot ou tout simplement à un enfant qui découvre la vie: l'usage de la fourchette pour manger, les dangers de l'alcool, la technique du pédalage... Un travail sur le matériau livré par les protagonistes permettra d'une part d'analyser les procédés rhétoriques utilisés dans la controverse, les figures de la preuve mobilisées dans leurs jugements, les quêtes de légitimité qui les animent. D'autre part, il nous sera possible d'étudier les principes moraux mobilisés. Enfin, en nous livrant les pistes pour remédier au dopage, leur vision de l'avenir, et leurs espoirs, ils expriment une visée politique particulièrement intéressante pour le sociologue.

\section{CONSTRUIRE UN CORPUS}

Un tel programme, permettant de renouer avec des traditions philosophiques sur le jugement, sur la morale et la politique, ne peut faire l'économie d'une méthodologie qu'il convient d'expliciter.

Avant tout, il convient de restreindre notre étude. La famille cycliste, considérée pendant longtemps comme une entité collective, ne cesse de se déchirer depuis l'édition 1998 du Tour de France (Duret et Trabal, 1999) ; l'ensemble des acteurs qui la composaient (leaders, équipiers, soigneurs, directeurs sportifs, organisateurs d'épreuve, et dans une certaine mesure, journalistes) a éclaté en autant de protagonistes impliqués 
dans une controverse publique qui constituera notre objet d'étude.

La crise consécutive à ce que les journalistes nomment «l'affaire Festina » ainsi que la publication par Menthéour (cycliste repenti), d'un premier livre sur la pratique du dopage dans le peloton, Secret Défonce, ont suscité de nombreux débats publics. L'écriture d'un livre constitue une forme de contribution à la polémique, qui a inspiré d'autres acteurs. Ce sont tout d'abord Nicolas Guillon et Jean-François Quénet qui vont publier les secrets du dossier Festina sous forme d'une enquête journalistique. Puis Willy Voet, le soigneur par lequel le scandale arriva, livrera une véritable confession avec son ouvrage Massacre à la chaîne: révélations sur 30 ans de tricherie. Mais en se confessant, il accuse: parmi les personnages soumis à sa vindicte, figure le très médiatique Richard Virenque. Contrairement à la plupart de ses ex-collègues, la ligne de défense du champion, a consisté, pendant longtemps ${ }^{1}$, à nier. Face à ces accusations, il lui faut donc s'expliquer et proclamer son innocence: Ma vérité, rédigé avec l'aide de deux « journalistesécrivains ", de son frère et de son avocat $\mathrm{M}^{\mathrm{e}}$ Collard, tentera de le disculper. Enfin, entendu dans le cadre de la mise en examen de Daniel Baal (président de la Fédération Française de Cyclisme), le directeur du Tour de France, Jean-Marie Leblanc ajoute à ces quatre ouvrages, une autobiographie réalisée sous la forme d'entretiens avec un journaliste, Christophe Penot, sous le titre Jean-Marie Leblanc gardien du Tour de France. Afin de garder une unité sur la forme de contribution au débat public, nous travaillerons donc à partir du corpus constitué par ces cinq livres. L'on pourrait objecter que la rédaction de ces textes est soumise à des contraintes éditoriales (notamment le recours à des « nègres » pour parfaire l'argumentation et le style). Dans ces conditions, il ne s'agirait plus du discours des acteurs mais de leur adaptation à des normes établies. Mais s'il semble difficile d'accéder aux processus d'écriture de ces livres, on peut cependant s'intéresser à ce que les acteurs acceptent de livrer puisque malgré tout ils ont fait leur ces narrations, en s'engageant par leur signature. Questionnaires, entretiens, articles les de presse, déclarations publiques, toutes les « données » discursives utilisées en sociologie sont liées aux contraintes de leur production. Les accepter ne revient pas à les nier mais à prendre acte que le discours est une activité intrinsèquement sociale.

Les « auteurs » du corpus peuvent être classés par leurs fonctions (deux cyclistes, un soigneur, un officiel, des journalistes) ou par leur rapport au dopage: deux repentis (Erwann Menthéour et Willy Voet), un accusé niant les faits ${ }^{2}$ (Richard Virenque), des accusateurs (les journalistes), un arbitre (Jean-Marie Leblanc). Mais il possible de distinguer ces ouvrages en prenant comme ligne de partage l'affichage ou non de révélations (《Secret défonce », « Massacre à la chaîne: révélations sur 30 ans de tricherie », «Les secrets» du dosser Festina »). Les deux derniers échappent à cette logique: «Ma vérité » est écrit comme une réponse à des accusations alors que celui sur J.M. Leblanc se présente comme une série d'entretiens. Une autre différence sensible sépare les genres d'écriture. A l'exception du livre de Nicolas Guillon et JeanFrançois Quénet construit comme un feuilleton présentant chronologiquement l'intégralité des épisodes de l'affaire Festina, les quatre autres textes présentent un point commun: le style biographique. Le mode de temporalité déployé par de tels récits et « l'exigence de vérité » (Ricœur, 1983) qui les accompagne vont renvoyer au statut du témoignage dans la quête épistémique de nos acteurs.

L'exigence pragmatique nous invitait à ne pas qualifier préalablement les acteurs. Les classifications précédemment mentionnées n'émanent pas d'un travail du sociologue mais bien des auteurs des livres. A ce titre, nous ne dérogeons pas à ce principe. Mais il ne saurait être question de poursuivre des opérations de qualification, notamment en recensant les positions des acteurs au moyen de connaissances externes (la proximité entre Leblanc et le groupe Amaury, le passé de tel ou tel

1. Ce n'est qu'en octobre 2000, lors du « procès Festina », qu'il avouera. Cet article, écrit avant cette date, ne prend pas en compte ce changement de stratégie.

2. Lors de la publication de son livre, il n'avait pas encore avoué. 
auteur, ses « réelles » motivations...). Il convient de prendre ces textes comme le résultat d'une action d'écriture, par laquelle les protagonistes clament publiquement leur « vérité » sur le dopage, en s'adressant à un lecteur «moyen » susceptible d'être convaincu, ému et indigné selon les mêmes principes qui les animent.

\section{TRAITEMENT DES DONNÉES}

Comment prendre en compte un tel corpus? Comment utiliser librement un outil qui rende compte des champs théoriques qui l'ont produit? Conçu et réalisé par Chateauraynaud et Jean-Pierre Charriau, le logiciel PROSPEROC, PROgramme de Sociologie Pragmatique Expérimentale et Réflexive sur Ordinateur, fut construit en tirant parti d'une confrontation des apports de la statistique textuelle (calculs des fréquences et de co-occurrences multiples), de la linguistique (analyse des énoncés et des enchaînements discursifs), de l'intelligence artificielle (système de raisonnement et d'inférence), des analyses de réseaux (calculs d'associations et de graphes) et de la sociologie cognitive (étude des procédés de codage et d'interprétation). Destiné à traiter d'importants corpus, il s'est enrichi en servant d'appui dans des études concernant les affaires, les controverses et tous les dossiers marqués par une grande diversité de formes de discours et pluralité des points de vue ou de styles d'argumentation. A ce titre, il semble le mieux adapté pour traiter l'ensemble de cinq ouvrages mentionnés, qui furent intégralement scannés.

Dans une première phase, le logiciel procède à l'étiquetage automatique des mots rencontrés dans le corpus: grâce à la forme des mots et aux répertoires construits lors des précédentes utilisations, il parvient à distinguer les entités (noms communs, noms propres, noms composés), des qualités (adjectifs et participes qualifiant les entités), des épreuves (principalement les verbes, conjugués ou non), des marqueurs (les adverbes, les expressions comme « de nouveau », « plus ou moins »...), des mots- outils (les pronoms, les conjonctions...) et des nombres. Ce traitement peut être corrigé manuellement et les mots non reconnus peuvent être classés. Prospéro permet de repérer des réseaux (en liant des mots comme « peloton », « équipe », « équipiers », « groupe »...), des figures de dénonciation (en groupant des mots comme «scandales », « salauds », « injustice», « mensonge »), des tendances à se justifier en référence au passé ou à l'avenir (grâce à des marqueurs comme « à l'époque », « dans les années », « ans plus tôt »). L'on fixe donc des catégories, des collections ${ }^{1}$ et des êtres-fictifs. La notion d'être fictif correspond à un noyau référentiel suffisamment stable pour que nos acteurs le mettent en scène, de façons différentes mais univoques (comme par exemple " le Tour », " le Tour de France », « la grande boucle »...).

Les principales différences de point de vue et d'argumentation s'expriment autour de trois axes: celui de la critique du jugement, celui de la critique de l'ordre moral et enfin celui de la critique politique. Par la critique du jugement, les auteurs posent la question de savoir comment distinguer le vrai du faux et y répondent en montrant en quoi leur parole ne peut être mise en doute. Ce processus d'authentification instaure le pacte de confiance indispensable entre le lecteur et son auteur. La deuxième visée de justification poursuivie par les auteurs est celle attestant de leur grandeur personnelle. Non seulement, ils disent la vérité, mais leurs prises de positions (et leurs actions) les élèvent. Le troisième thème auquel font référence les auteurs est celui de l'imputation de responsabilité (du dopage) à un niveau supérieur à celui des acteurs directement engagés.

\section{LA CRITIQUE DU JUGEMENT: CONVAINCRE DE LA VÉRACITÉS DE SES PROPOS}

Qu'il s'agisse des livres « révélation » (Menthéour, Guillon et Quénet, Voet), de celui sur Leblanc souhaitant s'expliquer sur son rôle, ou a fortiori celui de Virenque visant à «faire triompher la vérité », ces

1. Les collections sont des classes d'objets permettant d'objectiver les répertoires auxquels les acteurs font référence : par exemple, nous avons défini la collection des coureurs cyclistes (en saisissant la liste), la liste des produits dopants... 
textes cherchent à convaincre le lecteur de la véracité du propos. Aussi, avant même une analyse rhétorique des argumentations, il convient d'examiner les procédés utilisés pour emporter l'adhésion.

\subsection{Le récit à la première personne}

Dans ce type de narration, l'auteur se présente spontanément comme un témoin. En assumant un récit à la première personne, dans lequel on raconte avoir assisté, participé, observé, vu, touché quelque chose (Dulong, 1998), il se constitue comme dépositaire d'une preuve qui n'a pas besoin d'autre justification supplémentaire que la normalité du locuteur (Chateauraynaud 1996). Pour s'opposer à ce type de propos, l'on doit donc soit affirmer que le témoin est dépourvu des sens communs (on peut incriminer sa mauvaise vue ou sa folie - dans tous les cas sa normalité) soit le priver de sa qualité de témoin en l'accusant de menteur. En utilisant le « je » pour raconter un fait, l'auteur se présente avant tout comme un témoin direct des événements. Là où l'omerta pesait sur les pratiques de dopage, là où circulaient des rumeurs, des « on dit » qui nécessitent d'accorder sa confiance non seulement à celui qui rapporte l'histoire mais aussi à l'ensemble des personnes lui ayant rapportée, le témoignage direct offre des faits de «première main », avérés par un individu présent et identifiable. Dulong (1998) rappelle que le « j'y étais » nous fait adhérer spontanément au discours d'autrui. Il faudrait que dans le récit surviennent des faits incroyables et irréels pour qu'apparaissent le doute, ce qui en dehors d'histoires comiques, n'arrive presque jamais. Mais le statut du «j'y étais » lie le témoin à une position institutionnelle: le caractère irréfutable de son expérience singulière le soumet à réitérer « autant de fois qu'il le faudra, en toutes circonstances, face à toute contestation, la même version des faits. » (Ibid., pp. 165-166). Plus le récit biographique s'enrichit de détails cohérents avec ce que le lecteur connaît par ailleurs, plus sont rapportés des dialogues attestant non seulement de la présence physique du locuteur mais aussi de la charge émotionnelle accompagnant le contexte décrit, plus le lecteur est enclin à apporter sa confiance à l'auteur. Celle-ci se voit d'autant plus renforcée quand figurent des dialogues restituant avec une grande précision les phases de l'histoire et augmentant la tension dramatique par une accélération du récit.

A ce jeu, les propos de Menthéour et Voet apparaissent particulièrement habiles. Construit sur le mode de la confession, ce qui d'emblée leur évite d'avoir sans cesse à se donner le meilleur rôle (ils se présentent comme des personnages ayant parfois fauté), les récits favorisent d'autant plus la confiance du lecteur que sont décrites des pratiques, des savoir-faire, des procédés visant à faire face à des contrôles inopinés : comment pourrait-on inventer de tels détails qui supposent des pratiques habituelles inscrites dans la vie même du locuteur? Le « je » fonctionne comme un argument de poids et peut servir de caution tant que la parole de celui qui l'emploie ne peut être suspectée. On peut comprendre la force de persuasion du récit à la première personne en envisageant les conditions nécessaires pour ne pas y croire. Cette opération n'est pas qu'une spéculation: elle est imposée à ceux qui, accusés par des témoignages, veulent se défendre en niant les faits. C'est notamment la contrainte qui pèse sur Virenque: accusé par Voet, il doit « contrer Willy » (Virenque, p. 121). Sa dénonciation doit être imparable sans pour autant faire preuve de cruauté ou d'ingratitude envers celui qui se présente volontiers comme son père spirituel; elle ne doit pas en particulier transgresser un impératif de respect. Afin de préserver dans cette perspective son soigneur, sa stratégie consiste à imputer cette trahison à des tiers, ses avocats : « Manipulé, Willy qui est un type sensible s'est salement laissé embringuer dans une défense suicidaire »(Ibid., p. 123). L'argumentation de Virenque emprunte deux voies: s'en prendre au récit de Voet, s'en prendre à la personne de Voet. Mais elles sont peu compatibles car pour discréditer l'homme, il convient de montrer combien, calculateur et retors, Voet avait prémédité de tout noter sur ses fameux carnets. Cette passion de la mise en fiche suppose " des pratiques de deal brevetées » (Ibid., p. 121).

« Ces carnets, il les aurait tenus pendant dix-huit ans. Soigneusement, année par année? Il m'est alors permis de penser que ceux qui lui accordaient leur confiance s'étaient lourdement trompés » (Ibid.) 
L'inférence que Virenque demande implicitement à son lecteur donc est la suivante: vous avez une preuve que Voet en tenant des carnets m'a trahi et qu'il n'est pas digne de confiance. Pourquoi faire confiance aujourd'hui à son récit? Alors que pour s'en prendre au récit de Voet, Virenque tente de montrer également que le soigneur n'avait matériellement pas le temps de masser les coureurs et d'être présent durant les prétendues injections. Les deux arguments ne peuvent valoir en même temps; ou les injections ont bien eu lieu et Voet peut être accusé de traîtrise pour les avoir notées puis dévoilées (Virenque souligne notamment que le « traître » n'hésite pas à mettre en cause Jean-Claude Killy, Jean-Marie Leblanc, des sponsors, en un mot des figures de la famille cycliste - cf. Virenque, p. 122). Ou elles n'ont pas eu lieu et il peut être taxé d'affabulation, mais difficilement des deux à la fois. A trop vouloir prouver, l'argumentation perd de sa puissance. Ainsi, alors que la quantité de détails rend le récit biographique du soigneur plus réaliste, le nombre d'argumentations contradictoires pour le contrer affaiblit la stratégie du champion. C'est toute la différence entre un livre confession et un livre dénégation. Le logiciel PROSPEROC nous fournit le nombre d'occurrences de formes négatives utilisées dans le corpus; il est alors possible de différencier les auteurs ayant le plus recours à ces marqueurs. Après avoir ramené ce nombre sur une base de $100^{1}$, il apparaît que Leblanc et Virenque dépassent cette valeur (leur score est respectivement de 124 et 123) alors que Menthéour et Voet sont en dessous (respectivement 89 et 85); le livre des journalistes est quant à lui bien dessous, puisque son utilisation de formes négatives n'est crédité que de 74. Le fait que Leblanc et Virenque ont plus souvent recours à la dénégation que les autres auteurs du corpus souligne bien leur position d'accusé et l'exigence de réfutation qui les contraint. Les confessions publiques de Menthéour et Voet, conduisent inéluctablement à des accusations; il ne reste plus à ceux ainsi mis en cause, soit à avouer et à participer ainsi à un grand mouvement de conversion, soit à nier en apportant des preuves de leur innocence ou de leur bonne foi.

\subsection{Les figures classiques de la preuve et leurs limites}

La recherche de la vérité est aujourd'hui généralement fondée sur une quête de preuves scientifiques. S'adresser à l'autorité scientifique pour confirmer ou infirmer le recours à un produit dopant semble d'autant plus logique que ceux-ci ont été créés par des scientifiques. Leur incapacité à trancher de façon décisive est souvent dénoncée (ce sont les scientifiques qui sont à l'origine du dopage et il leur appartient de donner des éléments fiables pour l'éradiquer), parfois valorisée comme un exemple de prudence (contrairement à ceux qui accusent hâtivement sans se soucier de la faiblesse des preuves, les scientifiques n'hésitent pas à affirmer leur perplexité), toujours supposée entraîner l'émergence d'autres figures de la preuve. Même les appareillages les plus coûteux ne peuvent fournir que des paramètres indiquant une présomption ou une forte probabilité d'une pratique illicite et des indicateurs sujets à une discussion. La faiblesse - voire l'absence - de preuve scientifique conduit nos acteurs à arbitrer les controverses en mobilisant d'autres types de preuve. Les modalités de fonctionnement de la preuve journalistique dans les affaires de dopage ont déjà été étudiées (Duret, 1998, 1999). En procédant par recoupement d'indices, les journalistes se font un devoir de sortir l'enquête du laboratoire de la mener sur le terrain en faisant plus confiance à leurs yeux qu'aux résultats des analyses chimiques. L'accusation pour convaincre doit donc réussir à mettre bout à bout des signes épars, le cumul de plusieurs indices devenant, une fois réunis, accablants. Les plus frappants sont ceux pris sur le corps des dopés. Mais ils peuvent aussi bien se rechercher dans la vie des proches des athlètes; un entraîneur ou un conjoint dopé ou « dopeur » seront une source d'accusation. Enfin, l'amélioration des performances sera elle aussi suspectée dès qu'apparaissent des progressions aussi fulgurantes qu'inexplicables. L'introduction d'un nouvel acteur (judiciaire) modifie sensiblement les processus d'accusation.

1. Cette opération permet de tenir compte des tailles différentes des textes écrits par les auteurs et ainsi de neutraliser des variations directement liées à ces dimensions distinctes. 


\subsection{L'entrée de l'acteur judiciaire}

L'affaire Festina éclate suite à l'arrestation de Voet par des douaniers. Transmettant le dossier aux policiers du SRPJ puis au juge, ce sont de nouveaux acteurs qui s'emparent de la question du dopage: mais la quête de vérité du monde judiciaire s'appuie sur d'autres types de preuves. Les interrogatoires, le recoupement des témoignages, la mise sous dépôt des suspects permettant simultanément leur isolement et l'exercice d'une pression psychologique, favorisent l'aveu qui clôture - au moins provisoirement - le système de preuve. Doté d'une autorité indiscutable, le système judiciaire s'appuie parfois sur des preuves scientifiques (on n'hésite pas à confier des flacons à l'expertise scientifique), parfois sur des rumeurs (plusieurs acteurs rapportent que le contrôle de Voet n'était pas le fruit du hasard mais d'une délation), ou encore sur des évènements sportifs (il fallait profiter du Tour de France pour saisir le plus d'éléments possibles). Mais la qualification de la faute change: il ne s'agit plus de tricherie susceptible d'entraîner une suspension mais de transport « d'importation de substances vénéneuses » passible de prison ferme.

L'apparition de ce nouvel acteur judiciaire va désorganiser les procédés d'accusation et de justification. Tout d'abord, la réitération des aveux - répétition nécessaire pour tester la cohérence de la nouvelle version du récit - a conduit Menthéour puis Voet à publier des confessions. Cette forme de témoignage n'obéit pas à une contrainte policière mais vise au soulagement $\mathrm{du}$ poids d'une faute. Ici, en brisant la loi du silence, Menthéour comme Voet accusent tous les acteurs impliqués par le dopage et soumis à l'omerta; du coup, tant Virenque décrit comme l'un des principaux animateurs de l'approvisionnement en produits illicites chez Festina, que Jean-Marie Leblanc, « forcément dopé comme tout le monde » (Voet dans France Soir, 3 décembre 1998 - cité par Penot et Leblanc, p. 149), se trouvent soumis à une contrainte de justification. L'organisateur du Tour nie ce témoignage en invoquant sa simple présence et en retournant l'accusation: la responsabilité de ces vindictes publiques incomberaient à une intelligentsia pari- sienne [Penot et Leblanc, p. 149]. La justification de Virenque diffère de celle de Leblanc: face à l'existence des fameux carnets de Voet, il tente, nous l'avons vu, simultanément de trouver des failles dans le récit du soigneur ( « ces carnets sont des faux, crayonnés à la hâte pour détourner le feu » [Virenque, p. 122]) et de discréditer l'accusateur (la tenue de tels carnets « indique des pratiques de deal brevetées » [Ibid., p. 121]). Manifestement, ces carnets lui posent problème. Leur importance résulte elle aussi de la judiciarisation de la question du dopage. Là où les journalistes ne cherchaient que des traces de pilosité, où les scientifiques traquaient la présence d'un produit masquant, le pouvoir judiciaire tente de rassembler d'autres preuves tangibles: les bien nommées "pièces à conviction ». Les fameux carnets de Willy Voet soulignent que l'intrigue est plus policière et judiciaire que sportive. Les menaces dont le soigneur aurait fait l'objet suspendent provisoirement le versement de ces « pièces » au dossier. Reproduits dans son livre sur un fond noir, couleur utilisée dans le monde judiciaire pour mieux mettre en valeur ces preuves, ses carnets détaillent les doses de chaque produit administré aux coureurs de l'équipe Festina en utilisant un « code » : ce procédé renforce le sentiment de lire une intrigue policière sur le point d'être dénouée. Face à cette mise en scène dotée d'un pouvoir dramatique incontestable, l'iconographie utilisée dans le livre de Virenque apparaît comparativement plus faible: un album de photos, présenté sur fond bleu, vise à émouvoir le lecteur en s'appuyant sur des légendes expliquant la personnalité du coureur varois, ses joies et ses peines.

Enfin, l'entrée en scène de l'acteur judiciaire suscite une modification du rapport du monde sportif aux médias. Pendant longtemps, le spectre de la justice était agitée par des sportifs qui menaçaient de procès en diffamation les journalistes les accusant de dopage. Aujourd'hui les résultats des enquêtes judiciaires confortent une presse auto-érigée en victime de l'omerta. La couverture médiatique de l'enquête judiciaire apparaît à certains journalistes comme la possibilité d'une revanche. Leur travail évolue: alors qu'il s'agissait jusqu'ici de dénoncer quelques irrégularités 
pesant sur les valeurs incarnées par le modèle de l'épreuve sportive (Duret 1999, Yonnet 1998, Bromberger 1998...), l'ampleur de l'affaire génère un journalisme d'investigation (ce point suscite d'ailleurs un débat dans la profession - cf. Guillon, p. 108). A l'instar des policiers, les journalistes cherchent des traces. Des caméras montrent le contenus de poubelles laissées par une équipe lors du Tour 1998. Les témoignages sur le dopage sont recoupés avant d'être présentés à la personne interviewée - voir par exemple les relances de Christophe Penot (Penot et Leblanc, pp. 55, 114-115, 137, 149). Les sources sont systématiquement vérifiées et mises bout à bout [Guillon et Quénet]... Au même titre que les policiers et les juges qui parviennent à empêcher certains coureurs de disputer la suite du Tour (seul le pouvoir sportif, sur présentation d'un contrôle antidopage positif était jusqu'alors susceptible de le faire), des journalistes n'hésitent plus à peser sur le déroulement même de l'épreuve sportive: « Il faut arrêter ce Tour » demandait le Monde dans un éditorial (25 juillet 1998).

Virenque mène un travail de contreexpertise, d'enquête sur l'enquête journalistique, et montrant que les informations énoncées ne sont pourvoyeuses d'aucune véritable preuve [Virenque p. 10]. Mais Virenque n'est pas le seul à s'en prendre à la presse. Leblanc, bien que journaliste luimême, critique ses ex-confrères, pour les mêmes raisons: l'imprécision sert l'insinuation, réservoir où s'alimente la rumeur, mais pas la preuve [Penot et Leblanc, p. 223]. C'est bien le fonctionnement de la preuve journalistique qui est mise en cause: les recoupements d'indices, les inférences, les recherches de culpabilité diffèrent moins des méthodes judiciaires par leur procédés que par leur manque d'autorité institutionnelle. Aux accusations des journalistes, correspond symétriquement une dénonciation du mode de preuve qui fondent les enquêtes. Parallèlement, comme dans un souci de réflexivité, Virenque n'a de cesse de recourir très fréquemment à des figures d'argumentation ${ }^{1}$.

\section{CRITIQUE DE L'ORDRE MORAL : PRINCIPES ET FORMES DE LA GRANDEUR}

Les épreuves auxquelles sont soumis les coureurs ne sont pas toutes sportives. Il s'agit certes d'une part d'honorer leur contrat avec l'employeur en figurant en bonne place dans les courses afin que la marque soit associée à la performance voire à la victoire. D'autre part, il existe aussi des contrats moraux: s'engager vis-à-vis du public à assurer un beau spectacle (avec des attaques, des efforts pour contrer celles des adversaires, des contre-attaques...), agir simultanément dans le respect des lois de la famille cycliste en intégrant des valeurs comme la fidélité, la discrétion, la loyauté... Tenir ses engagements a un prix. Celui de l'effort pour figurer en tête d'une course suppose de " mouiller le maillot», « d'en baver », « de souffrir », de se « dépouiller» (Penot et Leblanc, p. 39)... En revanche, les valeurs morales qui font la famille cycliste demandent abnégation, flexibilité, sens du service. L'on perçoit qu'elles sont parfois incompatibles avec l'engagement vis-à-vis du public car les spectateurs sont demandeurs simultanément d'une éthique courbertinienne (une épreuve construite sur l'équité, la justice, le désintéressement au prix du labeur que rend bien l'expression « les forçats de la route ») et d'héroïsme (des sportifs assimilés à des Dieux, des géants survolant les épreuves, des sur-hommes nietzschéens).

\subsection{Premier principe: ne pas cesser de combattre}

La défaillance morale peut être interprétée dans le cadre de différentes grandeurs. Tout d'abord, il s'agit de ne pas

1. Si l'on distingue les auteurs faisant le plus usage d'expressions marquant une volonté de prouver (la liste des représentant de cette catégorie figure en annexe), l'ancien coureur de Festina arrive largement en tête. En ramenant, comme nous l'avons fait précédemment l'ensemble des occurrences à un indice neutre valant 100, le score de Virenque atteint 306, celui des journalistes 118, alors que les auteurs des ouvrages «confession» (Voet et Menthéour) obtiennent respectivement 38 et 16 . Ces valeurs renforcent l'idée selon laquelle, contrairement à ce qu'exige la justification, le style biographique de la confession est moins soumis à une contrainte de preuve. Notons par ailleurs, que les journalistes semblent se sentir très soumis à un impératif de preuve (leur score dépasse la valeur 100). 
« bâcher » c'est-à-dire abandonner dans le jargon cycliste (Penot et Leblanc, p. 97), mais tout autant de ne pas « en garder sous la pédale » (Virenque, p. 26) : une première grandeur renvoie donc au sens de l'effort. En repérant une catégorie d'entités bâtie autour de l'effort physique, l'on constate aussi que ce sont les deux cyclistes (Menthéour et Virenque) qui en font le plus usage (leurs scores relatifs respectifs sont de 206 et 136 alors les autres auteurs ont des scores inférieurs à 100).

Je ne l'ai pas volée. Je suis allé la chercher à la force du jarret. (...) Nous vivons une époque de communication dans laquelle l'image importe parfois plus que la performance. Mon image, je me la suis confectionnée à force d'embuscades, d'échappées sur des pentes de cols. (Virenque, pp. 26-27)

Issu d'un milieu modeste, il s'était hissé tout seul au sommet à la force de ses mollets et de son intelligence. (Menthéour, p. 67)

De même, la construction d'une catégorie d'épreuves rassemblant diverses modalités de la souffrance physique permet de noter la sur-représentation des expressions émanant des cyclistes: Menthéour, Virenque et Leblanc (une partie de l'ouvrage rappelle sa carrière sportive) obtiennent respectivement des scores relatifs de 138, 136 et 130 (alors que chez les autres auteurs, ils sont très inférieurs à 100).

\subsection{Deuxième principe: la fidélité à la famille}

Mais l'effort est loin d'être la seule grandeur à laquelle les acteurs font référence lorsqu'ils évoquent les notions d'engagement et d'ordre moral. Notamment, les sens de l'équipe, de la solidarité entre coureurs, de la famille (civile comme cycliste) constituent un ensemble de valeurs sur lesquelles les auteurs fondent leurs propos et leurs actions. Le grand nombre d'allusions à la cellule familiale (on ne dénombre pas moins de 605 occurrences) peut s'expliquer en partie par le style biographique auquel quatre des cinq auteurs du corpus ont recours. On note un grand nombre d'occurrences où le père, la mère ou le frère sont présentés comme ceux à qui on dédie la victoire, ceux vers qui on se tourne pour trouver de l'aide pour passer une épreuve difficile, ceux enfin qu'il convient d'honorer et surtout de ne pas déshonorer. L'ancrage familial de nos auteurs trouve son exacte transposition dans le mode de fonctionnement offert par la famille cycliste. Le passage « chez les pros » reste une expression marquant soit l'entrée dans le groupe (cf. par exemple Penot et Leblanc, p. 42, Virenque, p. 52), soit les frontières de cette famille par une description des usages le constituant (cf. par exemple Voet, p. 132-133). Le dopage, selon les repentis, figure parmi les pratiques faisant le groupe (cf. par exemple Menthéour p. 49, Guillon, p. 151). Le mécanisme décrit par Simmel (Simmel, 1991) dans le cas des sociétés secrètes permet de comprendre en quoi l'obligation de ne rien dire reste essentiellement fondée sur un contrat avec les autres membres: elle constitue un lien social positif fonctionnant sur une relation de réciprocité et de confiance entre les initiés. Les processus d'initiation, décrits notamment par Menthéour (Menthéour est, avec les journalistes, l'auteur qui obtient le plus haut score relatif à la catégorie "Secret/confidentialité »), s'apparentent à des renouvellements d'épreuves au cours desquelles, le candidat au peloton a l'occasion de quitter le monde des profanes en montrant qu'il accepte l'obligation de conserver le secret.

Il existe un rite initiatique du dopage. Tous les jeunes en entendent d'abord parler. Mais les anciens essaient de le cacher, pour entretenir la mystification. A partir du moment où il y a un interdit, et qu'on maintient le mystère autour, la tentation est grande de percer le secret pour entrer dans le cercle des initiés. (...) Le passage à l'acte se fait petit à petit. Cela commence souvent dans les courses par étapes, là où les efforts s'accumulent et où le moral du coureur, amateur ou professionnel, est soumis à des variations brutales. Il est éloigné de son milieu habituel, immergé dans la vie de son équipe. Il y a toujours quelqu'un, soigneur ou directeur sportif, pour lui « apprendre le métier » (Menthéour, pp. 58-59).

Les auteurs confessant la banalité du dopage, comme ceux le niant, s'accordent donc, malgré eux : les premiers soulignent sa capacité à créer un lien social dans la famille cycliste (mais ils la trahissent), les seconds regrettent son déchirement dont sont responsables les profanateurs. 
6.3 Troisième principe: la défense de la vérité

La trahison qui consiste à briser l'omerta peut être revendiquée au nom d'une autre grandeur: celle des repentis qui, préférant renoncer à la triche, se portent garants de la vérité. C'est le sens des introductions de Menthéour et de Voet: soumis à un impératif de fidélité à la famille cycliste (ils ont été initiés et à ce titre ont promis de se taire) et une volonté de trouver une sortie honorable (ils ont été reconnus fautifs), ils ont choisi de tout dire. La vérité ne supporte ni demimesure, ni concession: elle apparaît comme une cause dont chacun se réclame et pour laquelle on livre un combat sacré tout en la relativisant (les titres de Virenque et Menthéour évoquent leur vérité). Les jeux de polarisation vérité/mensonge, vrai/faux, exact/inexact renvoient à des oppositions fondant une grandeur morale séparant les honnêtes des malhonnêtes, et plus largement les victimes des tricheurs.

Être briefé ne signifie pas être initié à l'art de mentir. C'est simplement être averti de la façon dont nous allons être cuisinés. C'est une précision utile à apporter tant il a été dit que nous avions, et en particulier moi, été dressés à mentir pour notre défense. Toutes les allégations qui vont dans ce sens sont bien une manière entre mille autres de nous ridiculiser. Sinon de nous ridiculiser, en tout cas de nous détruire, de porter ombrage à notre probité. (Virenque, pp. 93-94)

\subsection{Quatrième principe: se conduire en homme}

Si l'aveu des pratiques illicites associé au statut de repenti se battant pour imposer la vérité permettent d'accéder à une certaine grandeur, le fait de se taire malgré les pressions physiques et psychologiques confère une autre forme de gloire. Les interrogatoires et les incarcérations lors des gardes à vue, apparaissent comme de véritables épreuves pouvant soit attester de la résistance du héros soit le conduire à sa faillite. L'épreuve judiciaire mobilise des qualités comparables à celles exigées lors de l'épreuve sportive, puisqu'il s'agit de résister tant physiquement à un moment difficile - il suffit de comparer la description de la chaleur par Menthéour lors d'une course (Menthéour, p. 120) et celle de
Virenque pendant sa garde à vue (Virenque, p. 96) - que psychologiquement en parant les coups que les adversaires tentent de porter au moral d'un coureur - on peut rapprocher de même le propos de Leblanc (Penot et Leblanc, p. 213) et celui de Virenque (Virenque, pp. 14-15). Le changement de statut le plus éprouvant pour le champion est occasionné par la grande chute du « héros » au « zéro ». Il touche en effet, dans un laps de temps réduit, les deux limites offertes à l'identité individuelle celle du «sur-homme » et celle du «soushomme ». D'abord adulé, assimilé à un demi-dieu, célébré pour sa capacité physique et psychologique à surmonter une épreuve sportive, la singularité du champion va presque jusqu'à faire douter de son humanité. Ensuite soumis à des fouilles anales, incarcéré avec la lie de la société (drogués, taulards, violeurs...), traqué jusque dans ses poubelles (Menthéour p. 178, Guillon et al., pp. 103 et 108), il va subir un traitement le propulsant à l'autre borne de l'humanité.

Nier en résistant à l'épreuve, ne pas craquer, ne pas vaciller. Ces contraintes semblent permettre de montrer que sa virilité notamment sa force physique et mentale sont à proprement parler, à toute épreuve. Il est capable de passer par-dessus, de passer outre cette expérience traumatisante. Craquer ou ne pas craquer apparaît comme l'ultime épreuve de celui qui veut montrer sa supériorité. La résistance au mauvais traitement policier renforce dans sa position de héros, celui qui s'en montre capable. Le brouillage des catégories opéré par la garde à vue où le coureur est traité comme n'importe quel autre suspect, échoue et devient alors objet de scandale. $\mathrm{Au}$ lieu de gommer les signes de la singularité, l'incarcération les conforte quand elle ne parvient pas à briser le champion. Le score relatif obtenu par Virenque à la catégorie « Craquer » explose puisqu'il obtient 684 (tous les autres auteurs ont des valeurs inférieures à 100).

\subsection{Cinquième principe: respecter l'em- ployeur}

On ne peut prétendre à une reconnaissance sans effort physique, nous l'avons dit. Mais l'effort, la souffrance physique et le 
dépassement ne peuvent se comprendre si l'on fait abstraction du contrat entre le coureur cycliste et son employeur. Négocié, explicite, écrit, ce contrat lie un sponsor et sportif. Cependant, si le prix de la sueur semble être convertible en valeur monétaire, le marché repose sur un troisième acteur: le public. La demande du sponsor est un effort non seulement physique, mais encore aisément perceptible par le plus grand nombre pour qu'il puisse en tirer les bénéfices. Dans ces conditions, la recherche d'une performance suffisamment médiatisable doit animer le coureur professionnel qui s'engage donc à faire du spectacle pour fournir une bonne publicité à la marque le soutenant. La tenue de ses engagements vis-à-vis de son sponsor figure donc parmi les contraintes morales pesant sur le cycliste professionnel. Mais à l'heure où l'on recherche les acteurs responsables du dopage, à l'heure où la marque Festina se fait connaître du grand public essentiellement grâce à l'affaire qui porte son nom, les sportifs demandent un autre statut: sans renoncer à la singularité de leur tâche qui exige intrinsèquement des efforts hors normes, ils revendiquent un statut associant la démesure de la performance à la banalité d'un métier honnête et rémunéré. «Que l'on nous laisse faire notre travail » est devenu le slogan repris par de nombreux coureurs lors du Tour 1998 (Duret et Trabal, 1999). Dans notre corpus, les notions d'engagement contractuel avec le sponsor, d'obligations pesant sur le coureur cycliste et des contraintes de la professionnalisation sont diversement abordés par nos auteurs.

C'est dans l'ouvrage de Virenque que l'on trouve le plus d'occurrences du mot « métier» (juste avant Leblanc, alors que les autres auteurs obtiennent des scores relatifs très inférieurs à 100). Dans la description que ce champion fait de son travail, il construit son image de vainqueur, de battant et lui adjoint une qualité indispensable à ses yeux, le respect vis-à-vis de l'employeur:

Dominateur, c'est un adjectif que j'admets sans problème. Et si, une fois de plus, certains s'en plaignent au sein du peloton, qu'ils s'interrogent sur le sens profond de leur métier, sur ce que posséder une licence professionnelle engage à être. (Virenque, p. 26)
Le cyclisme professionnel existe par la volonté des sponsors qui héritent, en cas de victoire ou d'héroïsme, de l'image monnayable de son ou de ses champions. Et, à ce jeu, il n'y a pas de philanthrope. Il faut des résultats. Une place chez les pros n'est jamais acquise. Il faut d'abord et avant tout se faire une place. Et, ensuite, la conserver. Oui, il existe un stress dans notre métier, celui de durer. (Virenque, p. 181)

Le temps du coureur s'oppose au temps du sponsor. Si les épreuves de l'employeur se déroulent sur une assez longue durée, celles de ces employés (surtout pour les sportifs) sont d'autant plus porteuses de tensions et d'enjeux, que la durée des contrats est courte.

[Roussel a] repensé à cette réponse de Miguel Rodriguez ${ }^{1}$ un jour où, inquiet, il l'interrogeait sur la poursuite du sponsoring de Festina: «Quand tu quitteras le vélo, moi, j'y serai toujours » (Guillon, p. 79).

Se posent alors inévitablement la question de la légitimité des contraintes pesant sur les cyclistes. Que se soit l'obligation de dopage si l'on en croit Menthéour (cf. notamment Menthéour, p. 59), ou la nécessité de préserver la santé des sportifs, les acteurs s'emploient à déterminer les responsabilités dans le dopage.

\section{CONCLUSION : VERS L'ANALYSE D'UNE CRITIQUE POLITIQUE}

Bien que vraisemblablement à l'origine des contrôles qui provoqueront l'affaire Festina, 1'Etat, construit sous Prospéro comme un être fictif, est un grand absent de notre corpus (il n'arrive qu'à la 81e place avec seulement 43 occurrences). Ce n'est pas tant le thème du dopage qui explique cette absence (l'analyse, avec les mêmes catégories, d'un corpus constitué des 1175 textes faisant apparaître le mot « dopage » dans les éditions du Monde datées entre 1987 et 1998, conduit à recenser quelques 1267 occurrences de cet être fictif, le plaçant au troisième rang des entités représentées) que les rhétoriques de nos auteurs. Cette impasse sur l'Etat provient plus des formes biographiques dont nous avons déjà évoqué l'intérêt qu'à une absence de discours politique. 
Tous les auteurs, même ceux qui nient l'existence du dopage, expriment des opinions, visant à rechercher des responsabilités, à dénoncer le fonctionnement de la justice, ou à proposer des pistes pour protéger les coureurs et lutter contre sa banalisation.

Une ligne de fracture très franche sépare les auteurs nous livrant leurs confessions (Menthéour et Voet) de ceux qui, sans nier que le dopage existe, affirment qu'il s'agit d'un problème marginal leur étant étranger (Leblanc, Virenque). Si les premiers tentent, tout en assumant leurs fautes, de généraliser le problème en évoquant le système du sport spectacle, les pressions économiques, les dérives de la rentabilité et du libéralisme, les seconds s'emploient à restreindre l'ampleur du dopage à des pratiques déviantes de quelques individus. Ils critiquent également les journalistes qui accentuent, à leurs yeux, excessivement et injustement le phénomène.

Politiser le débat sur le dopage permet à Menthéour et Voet, de restreindre leurs implications tout en les reconnaissant, de partir en quête de "vrais coupables », c'est-à-dire d'êtres, fictifs ou non, capable d'endosser de plus lourdes responsabilités, non transférables sur un tiers.

Il ne nous appartient pas de désigner qui, après les débats, est finalement le principal fautif. En revanche, il reste à rendre compte des processus mis en œuvre par les acteurs pour accuser et pour définir une politique permettant d'éradiquer le problème du dopage. L'on pourra alors recenser comment vont entrer dans l'arène, des nouveaux acteurs (ouvrant le débat à des négociations internationales sur la compatibilité des systèmes juridiques) et des nouvelles méthodes, notamment le fameux suivi médical longitudinal, qui fera l'objet de polémiques tant d'un point de vue scientifique (quels indicateurs retenir?), juridique (peut-on l'assimiler à une médecine du travail? Quel est le statut de l'employeur?) et moral (le secret médical doitil être préservé dans ce cas?). De même, il s'agira d'étudier les débats sur les poli- tiques sanitaires, sur la mondialisation et sur l'importance de l'argent, les auteurs du corpus n'hésitant à rapprocher la question $\mathrm{du}$ dopage d'autres questions sociales parmi lesquelles, la toxicomanie, le combat des opposants à Seattle, et le capitalisme.

Le dopage dans le cyclisme est source de bien d'incertitudes. Chaque épreuve en contient une part dont la somme constitue un enjeu central pour le spectacle sportif. A la forme traditionnelle du « scandale » reposant sur une réaffirmation unanime de la norme transgressée, par l'accusation d'un fautif bouc émissaire, se substitue aujourd'hui la forme « affaire » où cohabitent des sources d'indignations plurielles et contradictoires. Ajustements éthiques et transformations de ses règles ne s'opèrent plus seulement dans les coulisses de ses institutions. Les affaires sont cette mue qui se déroule sous nos yeux à la recherche de nouveaux interdits capables, après le professionnalisme et le dopage, de borner les frontières du mythe. La perte d'autonomie du mouvement sportif, longtemps satisfait de la politique de l'apolitisme, conduit ses institutions à un sentiment de dépossession (Waser, 2000, Defrance, 2000). En effet, Le scandale, opération d'épuration orchestrée de bout en bout par l'institution sportive la renforçait. Parce qu'elle lui échappe en partie l'affaire la menace plus directement. Les acteurs mis en cause peuvent à leur tour mobiliser des ressources juridiques, scientifiques ou médiatiques pour les retourner contre leurs accusateurs. Les affaires apprennent enfin, comment se transforment les normes sportives et comment se discutent les frontières entre le glorieux, le banal, le toléré et l'inadmissible. Rendues mouvantes, ces lignes de démarcation se redéfinissent quand se substitue à l'indignation unanime et sans appel un débat sur les causes mêmes de la transgression, mettant en intrigue des responsabilités incertaines plutôt que les désignant par avance. Du coup l'incertitude culmine, et c'est sans cette maximalisation de l'incertain qui explique l'engouement du public pour l'affaire ${ }^{1}$,

1.Le Monde du 21 juillet 1998 titre : «L'équipe bat des records de vente dans un climat de malaise». Les ventes des ouvrages constituant notre corpus sont assez éloquentes puisque le Monde du 28 mai 1999 parle de «la littérature sportive dopée à l'EPO» en soulignant qu'à cette date, le livre de Menthéour était déjà vendu à 90000 exemplaires alors que celui de Voet en était à sa «troisième réimpression atteignant le tirage exceptionnel de 122000 exemplaires ». 
comme nouvelle mise en intrigue du feuilleton sportif. Par delà l'incertitude liée au sort du champion, la question « fallait-il arrêter le tour 98 » résume bien l'incertitude ultime portant sur la mort de l'épreuve sportive elle-même, comme impossibilité de faire vivre une épreuve juste.

\section{BIBLIOGRAPHIE}

Benatouil, (1999).Critique et pragmatique en sociologie : quelques principes de lecture, Annales HSS, 2, 281-317

Boltanski, L. et Chiapello, E. (1999). Le nouvel esprit du capitalisme. Paris : Gallimard

Boltanski, L. et Thevenot, L. (1999). De la justification - les économies de la grandeur. Paris : Gallimard

Boltanski, L., Darre, Y., Schiltz, M.A. (1984). La dénonciation, Actes de la recherche en sciences sociales, 50, 2-40

Bourdieu, P. (1984). Questions de sociologie. Paris : Les éditions de Minuit.

Brohm, J. M. (1993). Les meutes sportives : critique de la domination, Paris : L'Harmattan

Bromberger, C. (1998). Football, la bagatelle la plus sérieuse du monde. Paris : Bayard Editions.

Chateauraynaud, F. (1998). Une sociologie pragmatique des alertes, in C. Gilbert et I. Bourdeaux (Coord.). Séminaire $d u$ Programme Risques Collectifs et Situations de Crise - Actes de la 11e séance. (pp.6282). Grenoble : Ecole Nationale Supérieure des Mines de Paris / C.N.R.S.

Chateauraynaud, F. (1996). Essai sur le tangible - entre expérience et jugement : la dynamique du sens commun et de la preuve. Ronéo EHESS. Paris.

Chateauraynaud, F. (1991). La faute professionnelle : une sociologie des conflits de responsabilité. Paris : Métailié.

Chateauraynaud, F. et Torny, D. (1999). Les sombres précurseurs : une sociologie pragmatique de l'alerte et du risque. Paris : Editions de l'Ecole des Hautes Etudes en Sciences Sociales.
Defrance, J. (2000) La politique de l'apolitisme : sur l'autonomisation du champ sportif, Politix, 50 : 13-27.

Dulong, R. (1998). Le témoin oculaire : les conditions sociales de l'attestation personnelle. Paris : Editions de l'Ecole des Hautes Etudes en Sciences Sociales.

Dulong, R. et al. (1999). Problèmes de la description et pratiques de la preuve. L'aveu comme fait juridique et comme phénomène moral. Rapport de recherche. Paris : E.H.E.S.S.

Duret, P., Trabal, P. (1999). Les cyclistes entre deux violences. Cultures en mouvement, $\mathbf{1 9}$, 44-50.

Duret, P. (1999). Juger les pratiques sportives. Esprit, 1, 92-109

Duret, P. (1998) Le tribunal des injustices. Communications, 67, 135-148.

Ehrenberg, A. (1991). Le culte de la performance. Paris : Calmann-Lévy.

Marchetti, D. (1998). Le football saisi par les médias, Société et représentation, 7, 309333.

Simmel, G. (1996). Secret et sociétés secrètes. ( $1^{\text {re }}$ édition : 1908). Strasbourg : Editions Circé.

Ricœur, P. (1983). Temps et Récit/ tome 1. Paris : Seuil

Yonnet, P. (1998). Systèmes des sports. Paris: Gallimard.

Waser, A.-M. (2000) De la règle du jeu au jeu avec la règle in F. Siri (Coord.) La fièvre du dopage. (pp. 187-199). Paris : Editions Autrement.

Les ouvrages du corpus :

Guillon, N. et Quenet J.-F. (1999) Les secrets du «Dossier Festina». Paris : Editions Solar.

Menthéour, E. (1999). Secret Défonce : ma vérité sur le dopage. Paris : Editions J.C. Lattès.

Leblanc, J.M., Penot C. (1999). Jean-Marie Leblanc, gardien du tour de France. SaintMalo : Editions Cristel.

Virenque, R. (1999). Ma vérité. Monaco : Editions du Rocher.

Voet, W. (1999). Massacre à la chaîne : révélations sur 30 ans de tricherie. Paris : Calmann-Lévy. 


\section{Doping im Profiradsport: Anklagen, Gestehen, Leugnen}

Zusammenfassung : Ziel dieses Artikels ist es, das Dopingproblem mittels der pragmatischen Soziologie zu untersuchen. Indem die Positionen der unterschiedlichen Protagonisten symmetrisch untersucht und ernst genommen werden, will dieser Ansatz die Bedeutung der politischen und moralischen Grundsätze der Akteure darlegen. Der Gegenstand des Artikels ist die Festina-Affäre bei der Tour de France 1998. Das Korpus, das auf fünf Büchern basiert (veröffentlicht von den Hauptakteuren), wurde mit dem Programm Prospéro untersucht. Wir untersuchten vor allem die von den Protagonisten benutzten Überzeugungsprozeduren, die „Größen“ auf die sie sich berufen, um ihre Konzeptionen von Gerechtigkeit und Ungerechtigkeit zu verteidigen, ebenso wie ihre politischen Positionen zur Zukunft des Radsports. Die „Figur“ des Berichts in der ersten Person, durch die sich der Sprecher als Zeuge präsentiert, der Fakten in Form von Geständnissen erzählt, erscheint als die wirksamste rhetorische Strategie. Wir haben 5 „Größen“ unterschieden, auf die sich die Protagonisten berufen, wenn sie auf Doping im Radsport zu sprechen kommen. Diese Pluralität erlaubt es Spannungen des Konflikts und die Gegensätze in ihren politischen Vorschlägen, um aus der Krise herauszukommen, zu verstehen.

Schlagwörter : Soziologie, Doping, Radsport, Gerechtigkeit, Beweis.

\section{Il doping nel ciclismo professionistico : accuse, confessioni e degenerazioni}

$\underline{\text { Riassunto: }}$ Lo scopo di quest'articolo è di studiare il problema del doping utilizzando gli apporti della sociologia pragmatica. Studiando simmetricamente le posizioni dei differenti protagonisti e prendendoli sul serio, questo approccio si propone di evidenziare l'importanza dei fondamenti politici e morali che guidano i protagonisti. L'affaire Festina del Tour de France 1998 costituisce l'oggetto di quest'articolo; il corpus, costituito partendo dai cinque libri (pubblicati dai principali protagonisti) fu analizzato con il programma Prospéro. In particolare abbiamo studiato le procedure utilizzate dai protagonisti per convincere, le "grandezze" alle quali si sono riferiti per difendere le loro concezioni del lecito e dell'illecito, così come i loro punti di vista politici sull' avvenire del ciclismo. La "figura" della recita alla prima persona, per la quale il locutore si presenta come un testimone che relaziona dei fatti sotto forma d'una confessione, appare come la più efficace delle strategie retoriche. Abbiamo distinto cinque "grandezze", alle quali i protagonisti si riferiscono quando evocano il doping nel ciclismo. Questa pluralità permette di comprendere le tensioni del conflitto così come le opposizioni nelle loro proposte politiche per uscire dalla crisi.

Parole chiave: ciclismo, doping, giustizia, prova, sociologia.

\section{El doping en el ciclismo profesional : acusaciones, confesiones, negativas}

Resumen: El objeto de este artículo es estudiar el doping a través de los aportes de la sociología pragmática. Estudiando de manera simétrica las diferentes posiciones de los protagonistas considerándolos de manera seria, esta aproximación se propone rendir cuenta de la importancia que tienen los fundamentos políticos y morales que guían a estos actores. El objetivo de este artículo es analizar el affaire Festina en el tour de Francia del año 1998 del corpus construido a partir de los cinco libros, es estudiado mediante el soft Prospero. Estudiamos los procesos realizados por los protagonistas que tratan de convencer, las dimensiones a las cuales se refieren por defender sus concepciones de legitimidad y de ilegitimidad, como también sus puntos de vistas políticos y el futuro del ciclismo. La figura del discurso en primera personal en la cual el locutor se presenta como un testigo relata los hechos sobre una forma de confesión, esto aparece como la más eficaz estrategia de retórica. Hemos distinguido cinco dimensiones en que los protagonistas se refieren al doping en el ciclismo. Esta pluralidad permite entender las tensiones del conflicto como también las oposiciones y sus proposiciones políticas que le permiten salir de la crisis.

Palabras claves : sociología, doping, ciclismo, justicia, pruebas. 